\title{
Bone Marrow-Derived Cells Contribute to Fibrosis in the Chronically Failing Heart
}

\author{
Po-Yin Chu, ${ }^{*}$ Justin Mariani, ${ }^{*}$ Samara Finch, ${ }^{*}$ \\ Julie R. McMullen, ${ }^{\dagger}$ Junichi Sadoshima, ${ }^{\ddagger}$ \\ Tanneale Marshall, ${ }^{*}$ and David M. Kaye* \\ From the Heart Failure Research Group," and Cardiac \\ Hypertrophy Laboratory, ${ }^{\dagger}$ Baker IDI Heart and Diabetes Institute, \\ Melbourne, Victoria, Australia; and the Departments of Cell \\ Biology and Molecular Medicine, ${ }^{\ddagger}$ New Jersey Medical School, \\ Newark, New Jersey
}

Cardiac fibrosis contributes significantly to the phenotype of the chronically failing heart. It is not clear whether in this setting the fibrosis is contributed by native cardiac fibroblasts or alternatively by recruitment of cells arising from the bone marrow. We aimed to determine the contribution of bone marrowderived cells to cardiac fibrosis in the failing heart and to investigate potentially contributing cytokines. Bone marrow-derived fibrocyte recruitment to the failing heart was studied in a transgenic (Mst1 mice) model of dilated cardiomyopathy. In conjunction, we examined the role of stromal-derived factor-1 (SDF1), a key chemoattractant, by assessing myocardial expression and secretion by cardiomyocytes and in clinical samples. Bone marrow-derived cells were recruited in significantly greater numbers in Mst1 versus control mice $(P<0.001)$, contributing $17 \pm 4 \%$ of the total fibroblast load in heart failure. Patients with heart failure had higher plasma levels of SDF-1 than healthy control subjects $(P<0.01)$. We found that cardiomyocytes constitutively secrete SDF-1, which is significantly up-regulated by angiotensin II. SDF-1 was shown to increases cardiac fibroblast migration by $59 \%(P<0.05)$. Taken together, our data suggest that recruitment of bone marrow-derived cells under the influence of factors, including SDF-1, may play an important role in the pathogenesis of cardiac fibrosis in heart failure. (Am J Pathol 2010, 176:1735-1742; DOI: 10.2353/ajpath.2010.090574)

Heart failure (HF) is a common, disabling clinical syndrome characterized by a constellation of symptoms and signs including breathlessness and exertional intolerance.
At the myocardial level, detailed echocardiographic, hemodynamic, and other imaging studies indicate that approximately half of patients presenting with HF have depressed ventricular systolic function, with the remainder seeming to have preserved systolic function. ${ }^{1}$ One commonality across both spectra of systolic and diastolic dysfunction is the finding of diffuse interstitial myocardial fibrosis, typically due to the deposition of collagens type I and III. ${ }^{2}$ Although myocardial fibrosis can have an impact on systolic function, its key sequelae on the myocardium is to increase ventricular stiffness, contributing substantially to the pathophysiology of $\mathrm{HF}^{3}$

In this context, it is widely acknowledged that myocardial fibrosis represents the balance of extracellular matrix deposition by myofibroblasts and extracellular matrix degradation by matrix metalloproteinases, which are in turn regulated by tissue inhibitors of the metalloproteinases. Traditionally, resident cardiac fibroblasts have been proposed to be the principal contributors to fibrosis, under the direction of paracrine and autocrine signals including angiotensin II, transforming growth factor $\beta$, endothelin, and connective tissue growth factor. ${ }^{2}$ On the other hand,other possible sources of cardiac fibroblasts have been proposed. It has been suggested that recruitment of circulating bone marrow-derived fibrocytes could contribute to the pool of fibroblasts, particularly in the context of ischemic damage. ${ }^{4}$ The paradigm of fibrocyte recruitment has also received particular attention in a number of noncardiovascular disorders including pulmonary fibrosis. ${ }^{5,6}$ In the cardiovascular context, in the setting of myocardial ischemia it is well known that the release of homing factors such as stromal-derived factor-1 (SDF-1) plays an important role in the trafficking of bone marrow-derived cells to the heart. ${ }^{7}$ However, the activity of this process in progressive heart failure, in the absence of acute ischemia, remains unknown. Other mechanisms that may contribute to the development of

Supported by the National Health and Medical Research Council of Australia.

Accepted for publication December 17, 2009.

Address reprint requests to Professor David M. Kaye, M.D., Ph.D., Heart Failure Research Group, Baker IDI Heart and Diabetes Institute, P.O. Box 6492, St. Kilda Rd. Central, Melbourne, VIC 8008, Australia. E-mail: david.kaye@ bakeridi.edu.au. 
fibrosis in the failing heart have also been proposed, including the derivation of cardiac fibroblasts by a process of endothelial to mesenchymal cell transition. ${ }^{8}$

On this basis of ongoing uncertainty about the mechanisms that cause myocardial fibrosis within the failing heart in the absence of acute myocardial ischemia, we aimed to investigate the hypothesis that heart failure drives a process of ongoing cardiac recruitment of bone marrow-derived circulating fibrocytes, similar to that suggested in pulmonary fibrosis. Such a mechanism would ultimately expand the pool of cardiac fibroblasts and thus myocardial fibrosis. Furthermore, we specifically investigated the role of SDF-1, given that its cognate receptor CXCR4 is known to be expressed on fibrocytes, ${ }^{9}$ and in so doing we aimed to investigate whether SDF-1 plays an important role in the pathogenesis of myocardial fibrosis. To address these questions we performed a complementary series using a mouse model (Mst1 transgenic) of dilated cardiomyopathy, isolated cardiomyocytes, and fibroblasts together with arterial and coronary sinus blood samples obtained from healthy subjects and patients with heart failure.

\section{Materials and Methods}

\section{Animals}

Twenty-four mice (12 Mst1 mice and 12 wild-type C57BL/6 mice) were used in our study. The Mst1 transgenic mice have been described in detail previously. ${ }^{10}$ In brief, Mst1 transgenic mice were generated on a C57BL/6 background, and Mst1 was overexpressed in a cardiac-specific manner using the $\alpha$-myosin heavy chain promoter. Wildtype C57BL/6 mice and green fluorescent protein (GFP)transgenic mice were obtained from Precinct Animal Centre (Baker IDI Heart and Diabetes Institute). All experimental procedures and protocols were approved by the AMREP Animal Ethics Committee, in accordance with the Guide for the Care and Use of Laboratory Animals published by the US National Institutes of Health. ${ }^{11}$

\section{Bone Marrow Transplantation and Fluorescence-Activated Cell Sorter}

Bone marrow cells were harvested from 6-week-old GFP transgenic mice. Twelve mice (six C57BL/6 and six Mst1 mice aged 6 weeks) received lethal irradiation with a total dose of 11 Gy (550 rad $\times 2$, separated by 3 hours). The remaining 12 mice did not receive irradiation. Unfractionated $\mathrm{GFP}^{+}$bone marrow cells $\left(5 \times 10^{6}\right.$ cells $)$ were resuspended in serum-free medium (Dulbecco's modified essential medium containing $0.5 \%$ penicillin-streptomycin and $1 \%$ glutamine). These cells were injected into the tail vein in a final volume of $0.2 \mathrm{ml}$. Mice were sacrificed at 13 weeks for assessment of cardiac fibrosis by Masson's trichrome staining and GFP cell accumulation. To assess chimerism, peripheral blood cells were collected from the recipient mice at the time of sacrifice, and the frequency of $\mathrm{GFP}^{+}$cells among peripheral nucleated blood cells was determined by fluorescence-activated cell sorter analysis using a FACS- can (BD Biosystems, Franklin Lakes, NJ). Analysis of flow cytometry data was performed using CellQuest Pro software (BD Biosciences, Franklin Lakes, NJ).

\section{Assessment of Myocardial Bone Marrow-Derived Cell Accumulation}

Mice were anesthetized with ketamine and xylazine, and the hearts were perfused with PBS solution and perfusion-fixed with $4 \%$ paraformaldehyde in PBS solution. Sliced hearts were then embedded (OCT compound, Miles Scientific, Naperville, IL) and quickly frozen in dry ice. Cryostat sections (5 $\mu \mathrm{m}$ thick) were stained with $H \& E$. Other serial sections that were stained overnight at $4^{\circ} \mathrm{C}$ with anti- $\alpha$-smooth muscle actin (SMA) (clone 1A4; Sigma-Aldrich, St. Louis, MO), anti-vimentin (clone GP53, Progen, Heidelberg, Germany), anti-collagen type I (Millipore Corporation, Billerica, MA), Alexa Fluor 647 anti-CCR7 (BioLegend, San Diego, CA), or anti-SDF-1 $\alpha$ (Fitzgerald Industries International, Concord, MA) were used to evaluate the mobilization, distribution, and localization of GFP ${ }^{+}$ $\alpha$-SMA ${ }^{+}, \mathrm{GFP}^{+}$vimentin ${ }^{+}, \mathrm{GFP}^{+}$collagen type $\mathrm{I}^{+}, \mathrm{CCR}^{+}$ collagen type $\mathrm{I}^{+}$, or SDF- $1 \alpha^{+}$cells. The sections were incubated for 1 hour at room temperature with secondary antibodies that had been conjugated (Alexa Fluor 546 or Alexa Fluor 647, Molecular Probes Inc., Eugene, OR). The nuclei were stained with Hoechst 33342 (Invitrogen Australia Pty. Ltd., Mount Waverley, VIC, Australia). Slides were observed under a fluorescent microscope. The GFP signal was confirmed by staining overnight at $4^{\circ} \mathrm{C}$ with either monoclonal (clone 1E4) or polyclonal anti-GFP (MBL Co., Ltd., Naka-ku Nagoya, Japan) antibodies. Morphometric analysis of H\&E-stained tissues was performed using ImagePro Plus software (Adept Electronic Solutions Pty. Ltd., Moorabin, VIC, Australia) at a magnification of $\times 400$. Fluorescent images were obtained using an Olympus Fluor Image Pro microscope. The quantification of $\mathrm{GFP}^{+}$cells was performed under a microscope (magnification $\times 400$ ). Ten fields of the heart tissue (left ventricle free wall, interventricular septum, and right ventricle free wall) were examined and were used to calculate the number of $\mathrm{GFP}^{+}$cells. Furthermore, the percentage of $\mathrm{GFP}^{+}$collagen type $\mathrm{I}^{+}$to collagen type $\mathrm{I}^{+}$was measured under a microscope (magnification $\times 400$ ). Ten fields of the heart tissue (left ventricle free wall) were examined and were used to calculate the number of $\mathrm{GFP}^{+} /$collagen I ${ }^{+}$cells.

Paraffin sections ( $4 \mu \mathrm{m}$ thick) were stained with Masson's Trichrome kit to evaluate the distribution and localization of collagen. The extent of fibrosis was quantitated in each of 10 randomly chosen fields per animal in different areas (left ventricle free wall, interventricular septum, and right ventricle free wall) using ImagePro Plus software (Adept Electronic Solutions Pty. Ltd. Perth, Australia) at a magnification of $\times 400$.

\section{Cell Culture Studies}

Neonatal rat ventricular cardiomyocytes were isolated from D1-3 Sprague-Dawley rats and cultured in six-well plates at a density of 250,000 cells per well as described 
previously. ${ }^{12}$ Two days after isolation cells were deprived of serum overnight. The following day cells were washed and placed in fresh Dulbecco's modified essential medium. Conditioned media were collected, as indicated, at various intervals for the determination of SDF-1. Cardiac fibroblasts were isolated as described previously ${ }^{13}$ and were used at passages three to six.

To determine the effect of SDF on fibroblast migration, $10^{5}$ cardiac fibroblasts (passages three to six) were seeded onto 24-well cell culture inserts (8- $\mu \mathrm{m}$ pore size, Transwell, Costar, Cambridge, MA). The lower chamber was filled with Dulbecco's modified essential medium alone or in conjunction with the agent of interest. Four hours after incubation, the number of cells that had traversed the insert membrane was determined by cell counting. In separate studies, the effect of SDF on protein synthesis was examined by measuring the rate of $\left[{ }^{3} \mathrm{H}\right]$ phenylalanine incorporation. In brief, cells were incubated for 6 hours in $\left[{ }^{3} \mathrm{H}\right]$ phenylalanine, followed by protein precipitation using tricarboxylic acid and subsequent measurement of radioactivity by liquid scintillation spectroscopy.

\section{Human Cardiac Catheterization Studies}

This study included 14 healthy volunteers and 29 patients with congestive heart failure (aged: $49 \pm 3$ versus $53 \pm 2$ years, respectively, $P=0.05$ ). Heart failure was due to nonischemic dilated cardiomyopathy in 16 patients and ischemic heart disease in 13 patients. The mean left ventricular ejection fraction was $24 \pm 2 \%$. All patients with $\mathrm{HF}$ received angiotensin-converting enzyme inhibitors, diuretics, and carvedilol. Healthy volunteers were recruited from the general community and were included in the absence of a history of cardiovascular disease, cardiovascular medications, and a normal cardiovascular examination. Patients with heart failure were recruited from the Alfred Hospital, Heart Failure Service. All studies were performed in the morning, and antifailure medications were continued to avoid hemodynamic instability. In patients with heart failure a balloon-tipped thermodilution catheter (7-Fr Arrow, Arrow International, Reading, PA) was inserted via an introducer sheath placed in the right internal jugular vein or antecubital vein, including the determination of pulmonary arterial pressures, wedge pressure, and cardiac output in the patients with HF. A right radial or brachial arterial line was placed for arterial blood pressure measurement and blood sampling. After the hemodynamic assessment a sampling catheter was positioned in the coronary sinus under fluoroscopic control. The tip of the catheter was positioned at least $2 \mathrm{~cm}$ proximal to the orifice of the coronary sinus, as confirmed by injection of radiographic contrast material. Arterial and coronary sinus blood samples were collected simultaneously to allow calculation of the transcardiac SDF-1 concentration gradient. After collection, blood samples were placed on ice. After the completion of the study, blood samples were centrifuged at $4^{\circ} \mathrm{C}$, and plasma was stored at $-70^{\circ} \mathrm{C}$ until subsequent assay. All patients gave written informed consent, and the study was per- formed with the approval of the Alfred Hospital Ethics Review Committee, in accordance with the Declaration of Helsinki.

\section{Biochemical and Molecular Biological Assays}

Plasma and conditioned media levels of SDF- $1 \alpha$ were determined by a commercially available enzyme-linked immunosorbent assay (R\&D Systems, Minneapolis, MN), according to the manufacturer's instructions. Real-time PCR to determine SDF mRNA expression was performed in ventricular cardiomyocytes. Total RNA was extracted using the TRIzol (Invitrogen, Carlsbad, CA) purification system and reverse-transcribed with TaqMan reagents (Applied Biosystems, Foster City, CA). Real-time PCR using a 12.5-ng template with an ABI Prism 7700 sequence detection system (Applied Biosystems) was conducted with the following primers: for rat SDF (forward) 5'-TGCCCCTGCCGATTCTT-3' and (reverse) 5'-GTGTTGAGGATTTTCAGATGTTTGA-3' and for the housekeeping gene GAPDH (forward) 5'-ACAGCAACTCCCACTCTTCC-3' and (reverse) 5'-CCTCTCTTGCTCAGTGTCC-3'. Expression values were determined by calculating the $\Delta \Delta \mathrm{Ct}$ value for each reaction, and data were expressed as fold difference compared with control.

\section{Statistical Analyses}

Values are presented as the mean \pm standard error of the mean. Between-group comparisons were performed using Student's $t$-test for normally distributed data. Comparisons between three or more groups were performed with analysis of variance. $P<0.05$ was considered significant.

\section{Results}

Thirteen-week-old Mst1 transgenic mice were characterized by extensive fibrosis in the left ventricle and right ventricle in comparison with wild-type mice (Figure 1, $A-E)$. The use of radiation per se did not alter the extent of myocardial fibrosis in either Mst1 mice of the C57BL/6 controls (data not shown).

\section{Bone Marrow-Derived Cells Are Recruited to the Nonischemic Failing Heart}

To determine whether bone marrow-derived cells are recruited to the failing heart, in the absence of myocardial injury due to ischemia or infarction, we developed chimeric mice by transplanting $\mathrm{GFP}^{+}$transgenic bone marrow cells into wild-type and heart failure (Mst1 transgenic) mice. We observed a similar degree of high-level GFP positivity in blood at 13 weeks in wild-type mice $(99.9 \%, n=3)$ and Mst1 transgenic mice $(94.8 \%, n=3$ each group) having undergone irradiation and bone marrow transplantation at 6 weeks of age. As expected, low-efficiency GFP expression was found in wild-type C57BL/6 (13 weeks, 18.5\%, $n=3$ ) and Mst1 (13 weeks, 
A
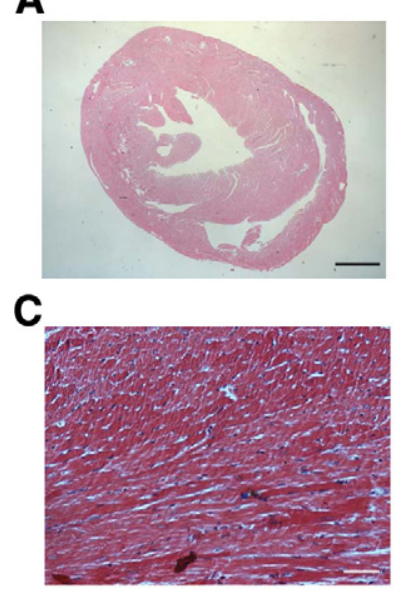

B

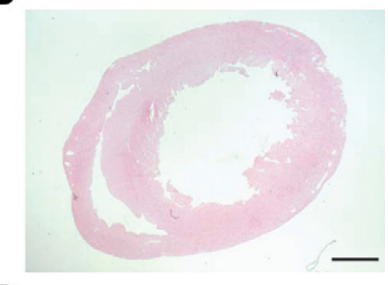

D

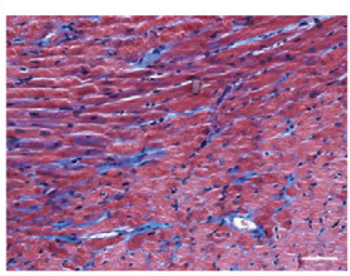

E

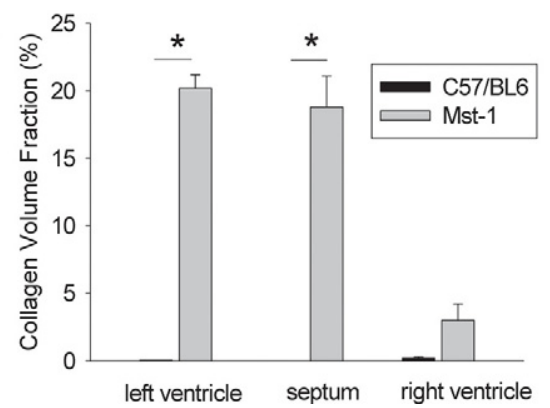

Figure 1. A and B: Low-power photomicrographs of H\&E-stained sections of $\mathrm{C} 57 \mathrm{BL} / 6$ (A) and Mst 1 transgenic (B) hearts from mice aged 13 weeks. Scale bars $=1 \mathrm{~mm}$. C and D: Photomicrographs of Masson trichrome-stained sections of $\mathrm{C} 57 \mathrm{BL} / 6(\mathbf{C})$ and $M s t 1$ transgenic $(\mathbf{D})$ mouse hearts. Scale bars = $50 \mu \mathrm{m}$. E: Bar graph representing collagen volume fraction in $\mathrm{C} 57 \mathrm{BL} / 6$ and Mst1 transgenic mouse hearts. ${ }^{*} P<0.01$.

$12.7 \%, n=3$ ) mice with bone marrow transplantation and no prior irradiation.

We next determined the number of $\mathrm{GFP}^{+}$cells in the myocardium of wild-type GFP-transplanted C57BL/6 mice and GFP-transplanted Mst1 mice at 13 weeks. As shown in Figure 2, A-C, the abundance of $\mathrm{GFP}^{+}$cells was significantly higher in the left ventricle of mice with dilated cardiomyopathy.

$\mathrm{GFP}^{+} \alpha-\mathrm{SMA}^{+}$cells, which are consistent with the identification of bone marrow-derived fibroblasts, were only observed in Mst1 mice (Figure 2D), in contrast with the GFP-transplanted C57BL/6 mice in which no such cells were identified. Likewise $\mathrm{GFP}^{+}$vimentin $^{+}$cells were also observed solely in Mst1 mice 13 weeks after irradiation and bone marrow transplantation, consistent with an origin of bone marrow-derived fibrocytic lineage (Figure $2 \mathrm{E}$ ). These cells were also shown to stain positively for collagen I, confirming their identity as fibroblasts (data not shown).

To investigate the relative contribution of bone marrowderived cell recruitment to the overall population of fibroblasts in the failing heart we determined the abundance of $\mathrm{GFP}^{+}$cells as a proportion of the overall number of fibroblasts. In control hearts minimal collagen staining was observed as indicated above by Masson's trichrome and by immunohistochemical analysis. Moreover no $\mathrm{GFP}^{+}$collagen I ${ }^{+}$cells were identified in C57BL/6 hearts. In Mst1 mice $16.9 \pm 4.4 \%$ of cells staining positive for
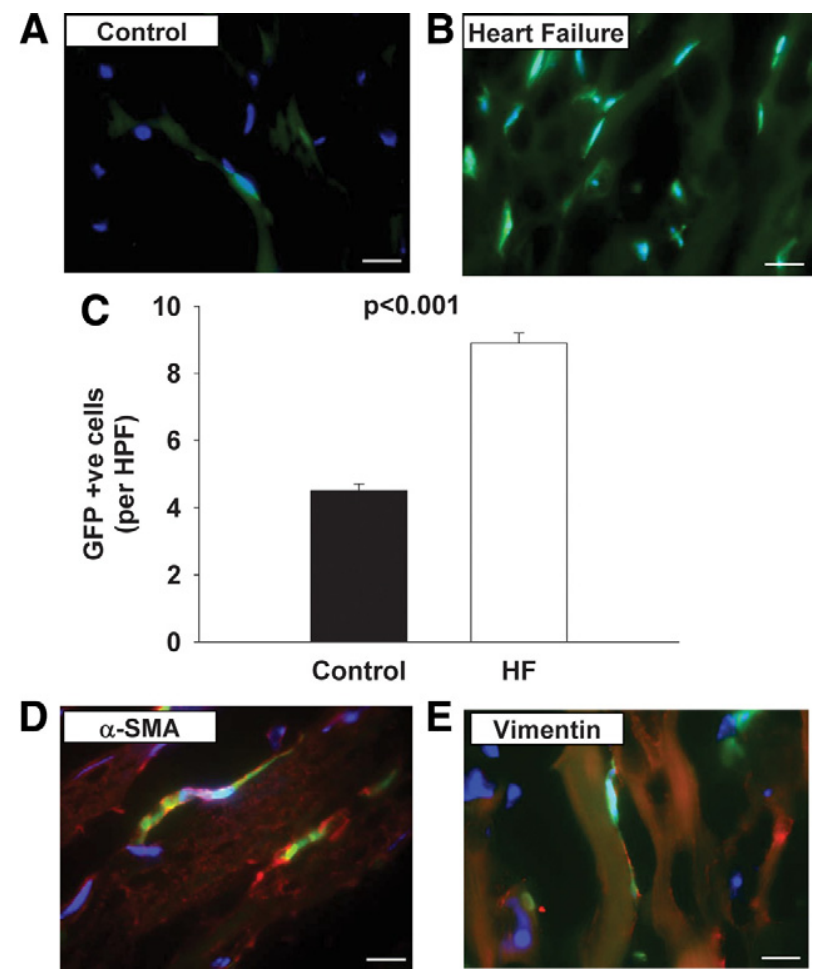

Figure 2. A and B: Merged fluorescence photomicrographs of sections of C57BL/6 (A) and Mst1 transgenic (B) mouse hearts demonstrating GFPpositive cells. Nuclear visualization was obtained by Hoechst 33342 staining. Scale bars $=10 \mu \mathrm{m}$. C: Bar graph showing the relative abundance of GFP-positive cells in C57BL/6 and Mst1 transgenic mouse hearts. HPF, high-power field. D and E: Dual fluorescence photomicrographs of sections from Mst 1 transgenic mouse hearts showing the presence of spindle-shaped GFP-positive cells staining for $\alpha$-SMA and vimentin, consistent with identification as fibrocytes. Nuclear visualization was obtained by Hoechst 33342 staining. Scale bars $=10 \mu \mathrm{m}$.

collagen I expressed GFP fluorescence. Taken together, these findings show that a significant fraction of fibroblasts in the myocardium of Mst1 mice arise from the bone marrow most likely via recruitment of circulating fibrocytes.

\section{SDF-1 Expression in Experimental Heart Failure}

Given that fibrocytes are known to express CXCR4, the receptor for SDF-1, we used immunohistochemistry to evaluate the myocardial expression of SDF- $1 \alpha^{+}$in Mst1 mice. SDF-1 expression was readily detected in Mst1 mice compared with that in C57BL/6 mice at 13 weeks (Figure 3, A and B). This result is consistent with the hypothesis that SDF-1 may be a key homing factor in the recruitment of bone marrow cells to the heart and thus may contribute to the pathogenesis of cardiac fibrosis in nonischemic cardiomyopathy.

\section{Peripheral SDF Levels and Myocardial SDF Production in Healthy Subjects and Subjects with Heart Failure}

The mean left ventricular ejection fraction of the HF cohort was $24 \pm 2 \%$ and the average New York Heart Associa- 
A

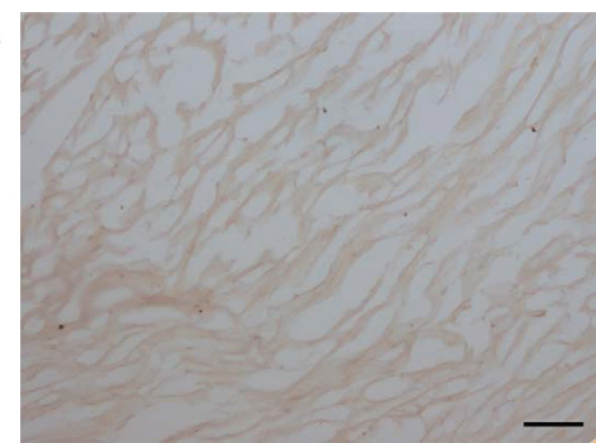

B
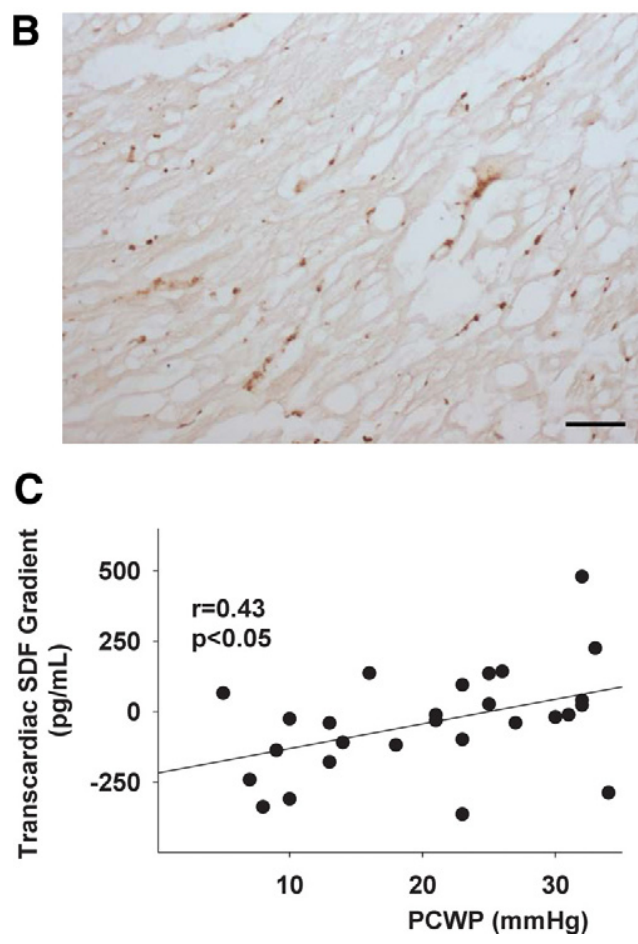

Figure 3. Photomicrograph of immunohistochemical sections stained for stromal-derived factor-1 expression in $\mathrm{C} 57 \mathrm{BL} / 6(\mathbf{A})$ and $M s t 1$ mice $(\mathbf{B})$. Scale bars $=10 \mu \mathrm{m}$. C: Line graph demonstrates the relations between the pulmonary capillary wedge pressure (PCWP) and transcardiac SDF-1 gradient in patients with heart failure.

tion heart failure class was $2.6 \pm 0.1$, consistent with moderate to severe heart failure. The mean right atrial pressure was $10 \pm 1 \mathrm{mmHg}$, the mean pulmonary capillary wedge pressure (PCWP) was $20.4 \pm 2.9 \mathrm{mmHg}$, and the mean cardiac index was $2.2 \pm 0.2 \mathrm{~L} / \mathrm{min} / \mathrm{m}^{2}$. The plasma arterial concentration of SDF-1 was significantly higher in patients with heart failure compared with control subjects $(2356 \pm 138$ versus $1709 \pm 113 \mathrm{pg} / \mathrm{ml}, P<$ 0.01 ). The net transcardiac gradient (plasma coronary sinus concentration - plasma arterial concentration) for SDF for the entire HF cohort tended to be higher than that in control subjects $(-31 \pm 34$ versus $-69 \pm 62 \mathrm{pg} / \mathrm{ml}$, NS). Despite a relatively uniform left ventricular ejection fraction, the patients with HF were characterized by substantial differences in their hemodynamic parameters. When classified according to the group mean PCWP, it was evident that subjects with high PCWP demonstrated net cardiac release of SDF, which was significantly different from that in the low filling pressure group $(21 \pm 45$
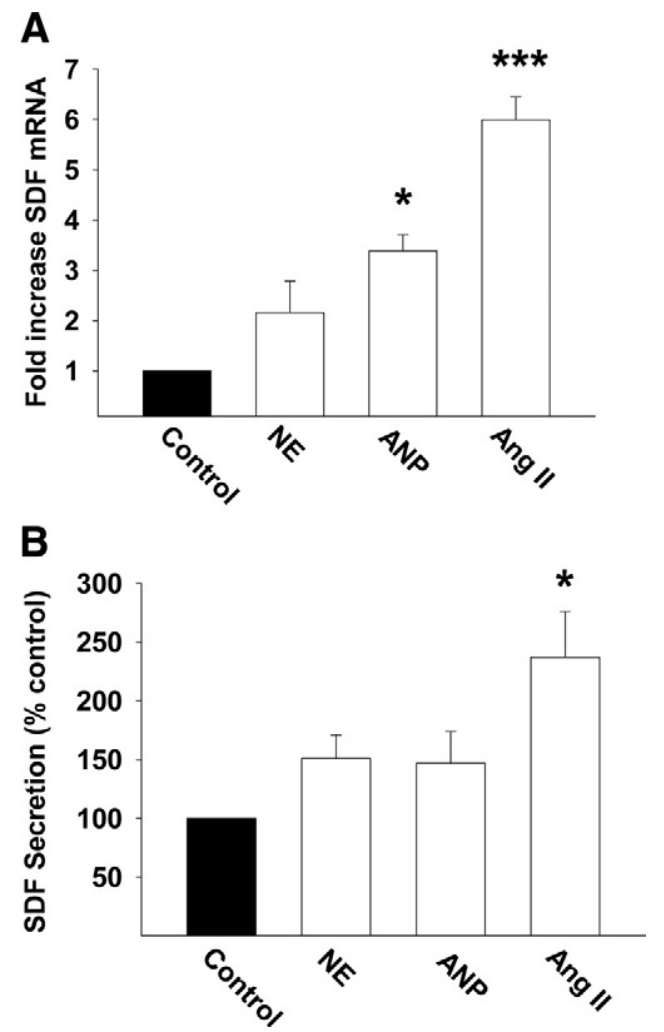

Figure 4. Bar graphs represent the influence of neurohormones (angiotensin II, norepinephrine, and atrial natriuretic peptide) on the expression of SDF-1 mRNA (A) and SDF-1 secretion $(\mathbf{B}) .{ }^{*} P<0.05 ;{ }^{* * * * *} P<0.001$

versus $-118 \pm 44 \mathrm{pg} / \mathrm{ml}, P<0.05)$. In the present study we did not detect a difference in the net release of SDF-1 according to the etiology of heart failure (ischemic versus nonischemic: $-27 \pm 38$ versus $-49 \pm 45 \mathrm{pg} / \mathrm{ml}$, NS), although this conclusion was limited by the relatively small sample size. In contrast, there was a significant correlation between the transcardiac SDF gradient and the PCWP $(r=0.43, P<0.05)$ (Figure 3C).

\section{Cardiomyocyte Secretion of SDF}

To assess whether cardiomyocytes exhibit the capacity to express and secrete SDF-1 we measured SDF-1 mRNA expression and protein secretion in cultured cardiac myocytes. SDF-1 mRNA was detectable in cardiomyocytes, and, in conjunction, we evaluated whether the expression of SDF-1 mRNA was modulated by neurohormones of relevance to HF. Angiotensin II caused a robust increase in SDF mRNA expression; atrial natriuretic peptide also caused a significant increase in gene expression, whereas norepinephrine was without influence (Figure 4A). Consistent with the effect on SDF gene expression, angiotensin II significantly increased the secretion of SDF-1 from cardiomyocytes, whereas norepinephrine and atrial natriuretic peptide were without effect (Figure 4B). 

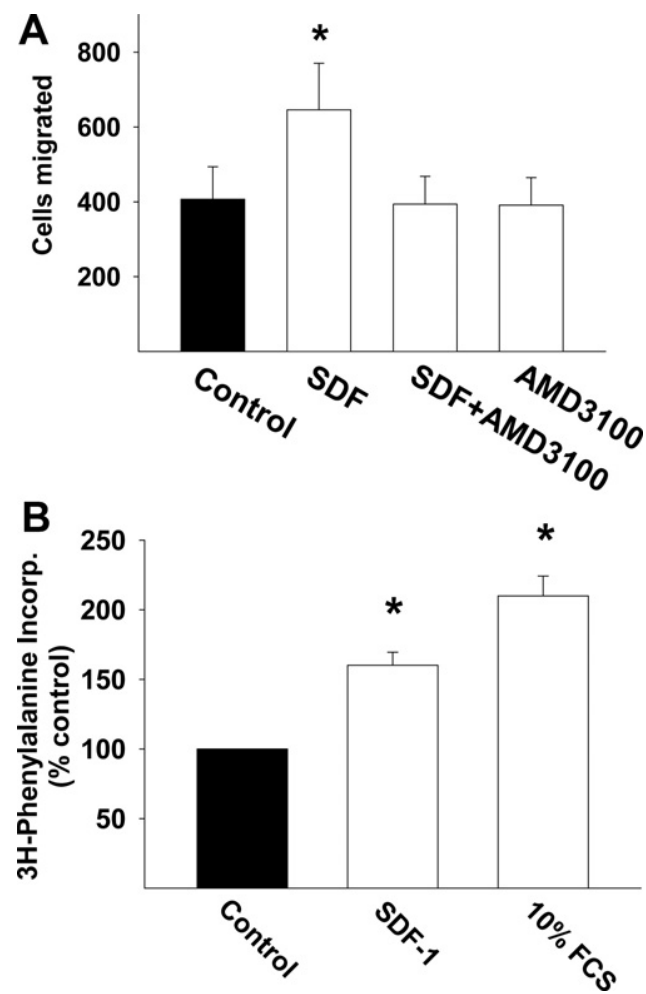

Figure 5. A: Bar graph demonstrates the effect of SDF-1 on fibroblast migration and its attenuation by the CXCR4 antagonist, AMD3100. B: Bar graph represents the effect of SDF-1 on fibroblast phenylalanine incorporation. ${ }^{*} P<0.05$.

\section{Influence of SDF on Cardiac Fibroblasts}

We next tested the hypothesis that locally secreted SDF could act as a homing factor for fibroblasts. As demonstrated in Figure 5A, $25 \mathrm{nmol} / \mathrm{L}$ exerted a robust effect on fibroblast migration $(P<0.05)$, similar in magnitude to that induced by $100 \mu \mathrm{mol} / \mathrm{L}$ angiotensin II (data not shown). Of note, this effect was abolished by the selective CXCR4 receptor antagonist, AMD3100. In parallel we determined whether SDF-1 increased the rate of protein synthesis in cardiac fibroblasts. As demonstrated in Figure 5B, SDF also significantly increased the rate of $\left[{ }^{3} \mathrm{H}\right]$ phenylalanine incorporation in fibroblasts.

\section{Discussion}

Cardiac fibrosis is a key determinant of the physiology of the myocardium in patients with symptoms of heart failure due either to systolic dysfunction or in this with heart failure with normal ejection fraction. Evidence for this association comes from histological analysis of both experimental and clinical heart failure samples ${ }^{14}$ or more recently from use of noninvasive imaging-based approaches in patients. ${ }^{15}$ In particular, the alteration in myocardial stiffness that results from myocardial fibrosis is brought about by a change in both the distribution and composition of the extracellular matrix. ${ }^{2}$ In this context, cardiac fibroblasts and their synthetic and migratory counterparts, the myofibroblasts, play a central role in determining the composition and rate of turnover of the extracellular matrix. Together with the rate of secretion of collagen and other matrix proteins, expression of the various matrix metalloproteinases and their regulators within the myocardium ultimately determines the extracellular composition. ${ }^{16}$

Given the critical role of the cardiac fibroblast in heart failure and thus for the development of antifibrotic strategies, it is important to understand the factors that determine the abundance and activity of these cells. Although it was previously considered that tissue fibrosis in general was the result of the activation and expansion of a pool of resident fibroblasts of mesenchymal origin, this concept has recently been challenged. Two hypotheses have been advanced, including the concept that resident cells of other lineage can differentiate into mesenchymal cells ${ }^{8}$ or that a pool of bone marrow-derived fibrocytes may accumulate in tissue in the presence of appropriate homing signals. ${ }^{6}$

We investigated the hypothesis that under the influence of a myocardial homing signal, bone marrow-derived fibrocytes accumulate in the setting of nonischemic cardiomyopathy. To date, studies of cell accumulation in the heart have typically used myocardial infarction or ischemia/reperfusion injury as the stimulus to show recruitment of bone marrow-derived cells, including fibrocytes to the heart. ${ }^{4}$ This paradigm is clearly different from that of chronic cardiomyopathy and to the best of our knowledge there have been no studies investigating whether recruitment of bone marrow cells to the heart occurs at all or what the potential attractants might be. In the present study, using the GFP-bone marrow transplant paradigm, we demonstrated for the first time that cells of bone marrow origin do accumulate in the Mst1 transgenic model of dilated cardiomyopathy and fully contribute to approximately one-fifth of the pool of fibroblasts in the failing fibrosed heart. We demonstrated that the GFP ${ }^{+}$ cells expressed a myofibroblast phenotype characterized by staining for $\alpha$-smooth muscle actin, vimentin, or collagen. Although our study provides strong evidence for bone marrow-derived cell accumulation in the failing heart as reflected by the accumulation of $\mathrm{GFP}^{+}$cells, it is appropriate to consider the potential limitations of GFP as a cell lineage marker. In particular, recent work ${ }^{17}$ has highlighted the potential limitations of this approach, including variable expression of GFP in different donor cells and the possibility that the local microenvironment could alter the expression of GFP by altered gene expression. To the best of our knowledge, fibrocyte recruitment to the failing heart in the nonischemic setting has not been previously documented. Previous work has shown that acute aortic banding in normal mice can elicit the expansion of fibroblasts of bone marrow origin within the heart, probably to some extent driven by a transforming growth factor- $\beta$-mediated process. ${ }^{8}$ The Mst 1 transgenic model of dilated cardiomyopathy is based on the cardiac-specific overexpression of Mst1, a member of the STE20 kinase family, which plays a role in the mediation of apoptosis. ${ }^{1}$ Although apoptosis has also been demonstrated to be relevant to the pathogenesis of human heart failure, it is not clear whether Mst1 per se plays a role in such a process. 
The renin-angiotensin-aldosterone axis has been ascribed a pivotal role in the pathogenesis of cardiac fibrosis in a range of cardiovascular disease states ranging from hypertension to heart failure. In particular, cell culture studies of angiotensin II have suggested that it plays a lead role as a mediator in the pathogenesis of fibrosis by promoting the differentiation of fibroblasts into myofibroblasts via a variety of signaling pathways. ${ }^{19,20}$ Interestingly, however, the evidence that angiotensin II acts alone to elicit cardiac fibrosis is not compelling. In mice subjected to pressure overload the extent of cardiac fibrosis was not mitigated in animals with angiotensin receptor type la knockout. ${ }^{21}$ Aldosterone has also been proposed to exert profibrotic effects, although its extent and mechanism remain controversial. ${ }^{22}$

Fibrocytes are bone marrow-derived cells that posses the potential to enter tissue and transition into fibroblasts, expressing $\alpha$-smooth muscle actin, collagen type I, and collagen type III. ${ }^{23}$ In the present study we did not specifically establish whether circulating bone marrow-derived cells with a fibrocytic phenotype were detectable in blood from animals with heart failure. Given the demonstration of recruitment of fibrocytes to failing myocardium, we next considered the identity of relevant chemoattractants. Fibrocytes have been demonstrated to express a variety of chemokine receptors including CCR3, CXCR4, CCR5, and CCR7. ${ }^{9,24}$ Of these chemokines, CXCR4, the cognate receptor for SDF-1, has received particular attention with regard to its role as a putative chemoattractant in the ischemic myocardium, ${ }^{7}$ most notably in regard to the recruitment of bone marrow-derived cells. In their study, Abbott et al ${ }^{7}$ showed that antagonism of SDF-1 in the setting of cardiac injury due to ischemia reduced the recruitment of bone marrow cells in support of our study.

Cardiac fibroblasts have been previously shown to express both CXCR4 and SDF-1. ${ }^{25}$ In the present study we demonstrated that there was a diffuse increase in the expression of myocardial SDF-1 protein in mice with heart failure. To extend these observations further, we measured the arterial and transcardiac gradient in normal healthy individuals and those with heart failure. The peripheral plasma arterial concentration of SDF-1 was significantly higher in patients with HF. In regard to the heart specifically, we observed that in healthy individuals and patients with well controlled heart failure, there was no net cardiac release of SDF-1. However, in patients with severe heart failure and markedly elevated filling pressures there was net release of SDF-1. Although this observation does not establish a direct causal link, we have recently shown that the extent of cardiac fibrosis progressively increases as the severity of HF advances using magnetic resonance imaging. ${ }^{14}$ In the present study we did not perform MRI routinely as several patients already had implanted pacemaker defibrillators, and echocardiographic measures of diastolic function were not possible in a number of patients because atrial fibrillation was present.

Thus, expression of SDF-1 at levels resulting in net release from the heart might be expected to drive the recruitment of bone marrow-derived cells expressing CXCR4 receptors, including fibrocytes. Of interest, it has been previously shown that the progression of heart failure is more malignant in patients in whom the filling pressures remain substantially elevated ${ }^{26}$ potentially because of the ongoing perpetuation of a profibrotic process such as that identified here. In support of this concept, we show that SDF- 1 is a significant chemoattractant for cardiac fibroblasts. This concept is discordant with a study by Abbott et al, ${ }^{7}$ who suggested that myocardial SDF expression alone is not sufficient to recruit stem cells. This study, however, was conducted in murine normal hearts in which SDF was only transiently overexpressed via gene delivery. In our study we examined the development of fibrosis and recruitment of $\mathrm{GFP}^{+}$cells over a prolonged period, which is a much more consistent time span relevant to the pathophysiology of heart failure. Other studies ${ }^{25,27}$ have suggested that SDF may be protective in the setting of ischemia-reperfusion injury either by promoting recruitment of angiogenic or progenitor stem cells.

Our studies clearly showed that the failing myocardium and cardiomyocytes express and can secrete SDF. Although in the normal human heart we did not detect "release" of SDF, it is relevant to consider that net release represents the combination of local release and extraction (including binding and degradation). Myocardial SDF-1 levels are known to increase after myocardial infarction, with concomitant increases in the rate of bone marrow-derived cardiac progenitor cells. ${ }^{28}$ In the present study we showed that angiotensin II promoted the secretion of SDF- 1 by cardiac myocytes. Previously it has been shown that angiotensin II exerts a pro-angiogenic effect, possibly mediated by factors such as vascular endothelial growth factor and basic fibroblast growth factor. ${ }^{29,30}$ Moreover, myocardial stretch is known to result in the release of growth factors and hormones, including angiotensin II, from cardiomyocytes, perhaps contributing to autocrine myocardial release of SDF-1. ${ }^{31-33}$

In the present study we aimed to evaluate the role of SDF-1 as a contributor to myocardial fibrosis in the setting of chronic heart failure, which has not been studied previously. Our data support the notion that elevated SDF-1 expression may contribute to bone marrow cell recruitment and fibrosis of the failing heart. We did not directly test the effect of SDF-1 antagonism in our murine model to demonstrate the amelioration of fibrocyte recruitment; however, our findings are consistent with previous studies in which SDF-1 expression was altered by acute ischemia. In the context of heart failure we demonstrate SDF-1 release by isolated cardiomyocytes in the presence of neurohormonal stimulation and from the failing human heart. Finally we documented a chemoattractant effect of SDF-1 on cardiac fibroblasts. These observations, therefore, lay the foundation for the further development of antifibrotic strategies in heart failure.

\section{Acknowledgment}

We gratefully acknowledge the expert technical assistance of Mr. Peter Kanellakis. 


\section{References}

1. Hogg K, Swedberg K, McMurray J: Heart failure with preserved left ventricular systolic function; epidemiology, clinical characteristics, and prognosis. J Am Coll Cardiol 2004, 43:317-327

2. Berk BC, Fujiwara K, Lehoux S: ECM remodeling in hypertensive heart disease. J Clin Invest 2007, 117:568-575

3. Feldman AM, Li YY, McTiernan CF: Matrix metalloproteinases in pathophysiology and treatment of heart failure. Lancet 2001, 357:654-655

4. Haudek SB, Xia Y, Huebener P, Lee JM, Carlson S, Crawford JR, Pilling D, Gomer RH, Trial J, Frangogiannis NG, Entman ML: Bone marrow-derived fibroblast precursors mediate ischemic cardiomyopathy in mice. Proc Natl Acad Sci USA 2006, 103:18284-18289

5. Phillips RJ, Burdick MD, Hong K, Lutz MA, Murray LA, Xue YY, Belperio JA, Keane MP, Strieter RM: Circulating fibrocytes traffic to the lungs in response to CXCL12 and mediate fibrosis. J Clin Invest 2004, 114:438-446

6. Strieter RM, Gomperts BN, Keane MP: The role of CXC chemokines in pulmonary fibrosis. J Clin Invest 2007, 117:549-556

7. Abbott JD, Huang Y, Liu D, Hickey R, Krause DS, Giordano FJ: Stromal cell-derived factor- $1 \alpha$ plays a critical role in stem cell recruitment to the heart after myocardial infarction but is not sufficient to induce homing in the absence of injury. Circulation 2004, 110: 3300-3305

8. Zeisberg EM, Tarnavski O, Zeisberg M, Dorfman AL, McMullen JR, Gustafsson E, Chandraker A, Yuan X, Pu WT, Roberts AB, Neilson EG, Sayegh $M H$, Izumo S, Kalluri R: Endothelial-to-mesenchymal transition contributes to cardiac fibrosis. Nat Med 2007, 13:952-961

9. Gomperts BN, Strieter RM: Fibrocytes in lung disease. J Leukoc Biol 2007, 82:449-456

10. Yamamoto S, Yang G, Zablocki D, Liu J, Hong C, Kim SJ, Soler S, Odashima M, Thaisz J, Yehia G, Molina CA, Yatani A, Vatner DE, Vatner SF, Sadoshima J: Activation of Mst1 causes dilated cardiomyopathy by stimulating apoptosis without compensatory ventricular myocyte hypertrophy. J Clin Invest 2003, 111:1463-1474

11. Institute of Laboratory Animal Resources: Guide for the Care and Use of Laboratory Animals, 7th ed. Institute of Laboratory Animal Resources, Commission on Life Sciences, National Research Council, Washington, DC, 1996

12. Simpson P: Stimulation of hypertrophy of cultured neonatal rat heart cells through an $\alpha 1$-adrenergic receptor and induction of beating through an $\alpha 1$ - and $\beta 1$-adrenergic receptor interaction. Circ Res 1985, 56:884-894

13. Stewart AF, Rokosh DG, Bailey BA, Karns LR, Chang KC, Long CS, Kariya K, Simpson PC: Cloning of the rat $\alpha_{1 \mathrm{C}}$-adrenergic receptor from cardiac myocytes. $\alpha_{1 \mathrm{C}}, \alpha_{1 \mathrm{~B}}$, and $\alpha_{1 \mathrm{D}}$ mRNAs are present in cardiac myocytes but not in cardiac fibroblasts. Circ Res 1994, $75: 796-802$

14. Weber K, Brilla C, Janicki J: Myocardial fibrosis: functional significance and regulatory factors. Cardiovasc Res 1993, 27:341-348

15. Iles L, Pfluger H, Phrommintikul A, Cherayath J, Aksit P, Gupta SN, Kaye DM, Taylor AJ: Evaluation of diffuse myocardial fibrosis in heart failure with cardiac magnetic resonance contrast-enhanced T1 mapping. J Am Coll Cardiol 2008, 52:1574-1580

16. Nagase H, Visse R, Murphy G: Structure and function of matrix metalloproteinases and TIMPs. Cardiovasc Res 2006, 69:562-573

17. Swenson ES, Price JG, Brazelton T, Krause DS: Limitations of green fluorescent protein as a cell lineage marker. Stem Cells 2007, 25:2593-2600
18. Dan I, Watanabe NM, Kusumi A: The Ste20 group kinases as regulators of MAP kinase cascades. Trends Cell Biol 2001, 11:220-230

19. Booz GW, Baker KM: Molecular signalling mechanisms controlling growth and function of cardiac fibroblasts. Cardiovasc Res 1995 30:537-543

20. Shen WL, Gao PJ, Che ZQ, Ji KD, Yin M, Yan C, Berk BC, Zhu DL $\mathrm{NAD}(\mathrm{P}) \mathrm{H}$ oxidase-derived reactive oxygen species regulate angiotensin-II induced adventitial fibroblast phenotypic differentiation. Biochem Biophys Res Commun 2006, 339:337-343

21. Harada K, Komuro I, Shiojima I, Hayashi D, Kudoh S, Mizuno T, Kijima K, Matsubara H, Sugaya T, Murakami K, Yazaki Y: Pressure overload induces cardiac hypertrophy in angiotensin II type $1 \mathrm{~A}$ receptor knockout mice. Circulation 1998, 97:1952-1959

22. Young MJ, Lam EY, Rickard AJ: Mineralocorticoid receptor activation and cardiac fibrosis. Clin Sci (Lond) 2007, 112:467-475

23. Quan TE, Cowper S, Wu SP, Bockenstedt LK, Bucala R: Circulating fibrocytes: collagen-secreting cells of the peripheral blood. Int J Biochem Cell Biol 2004, 36:598-606

24. Abe R, Donnelly SC, Peng T, Bucala R, Metz CN: Peripheral blood fibrocytes: differentiation pathway and migration to wound sites. J Immunol 2001, 166:7556-7562

25. Hu X, Dai S, Wu WJ, Tan W, Zhu X, Mu J, Guo Y, Bolli R, Rokosh G Stromal cell derived factor $-1 \alpha$ confers protection against myocardia ischemia/reperfusion injury: role of the cardiac stromal cell derived factor-1 $\alpha$ CXCR4 axis. Circulation 2007, 116:654-663

26. Stevenson LW, Tillisch JH, Hamilton M, Luu M, Chemilsky-Fallick C Moriguchi J, Kobashigawa J, Walden J: Importance of hemodynamic response to therapy in predicting survival with ejection fraction $\leq 20 \%$ secondary to ischemic or non-ischemic dilated cardiomyopathy. Am J Cardiol 1990, 66:1348-1354

27. Haider H, Jiang S, Idris NM, Ashraf M: IGF-1-overexpressing mesenchymal stem cells accelerate bone marrow stem cell mobilization via paracrine activation of SDF- $1 \alpha / C X C R 4$ signaling to promote myocardial repair. Circ Res 2008, 103:1300-1308

28. Kucia M, Dawn B, Hunt G, Guo Y, Wysoczynski M, Majka M, Ratajczak J, Rezzoug F, Ildstad ST, Bolli R, Ratajczak MZ: Cells expressing early cardiac markers reside in the bone marrow and are mobilized into the peripheral blood after myocardial infarction. Circ Res 2004, 95:1191-1199

29. Chua CC, Hamdy RC, Chua BH: Upregulation of vascular endothelial growth factor by angiotensin II in rat heart endothelial cells. Biochim Biophys Acta 1998, 1401:187-194

30. Pupilli C, Lasagni L, Romagnani P, Bellini F, Mannelli M, Misciglia N Mavilia C, Vellei U, Villari D, Serio M: Angiotensin II stimulates the synthesis and secretion of vascular permeability factor/vascular endothelial growth factor in human mesangial cells. J Am Soc Nephrol 1999, 10:245-255

31. Kaye D, Pimental D, Prasad S, Maki T, Berger HJ, McNeil PL, Smith TW, Kelly RA: Role of transiently altered sarcolemmal membrane permeability and basic fibroblast growth factor release in the hypertrophic response of adult rat ventricular myocytes to increased mechanical activity in vitro. J Clin Invest 1996, 97:281-291

32. Campbell D, Aggarwal $\mathrm{A}$, Esler M, Kaye D: $\beta$-Blockade reduces angiotensin II levels in heart failure patients receiving ACE inhibitor therapy. Lancet 2001, 358:1609-1610

33. De Boer RA, Pinto YM, Van Veldhuisen DJ: The imbalance between oxygen demand and supply as a potential mechanism in the pathophysiology of heart failure: the role of microvascular growth and abnormalities. Microcirculation 2003, 10:113-126 